\title{
Ribavirin derivatives with a hexitol moiety: synthesis and antiviral evaluation
}

\author{
Arthur Van Aerschot ${ }^{1 *}$, Guy Schepers ${ }^{1}$, Roger Busson ${ }^{1}$, Jef Rozenski ${ }^{1}$, Johan Neyts ${ }^{2}$, Erik De Clercq ${ }^{2}$ \\ and Piet Herdewijn ${ }^{1}$
}

'Laboratory of Medicinal Chemistry and 'Laboratory of Virology and Chemotherapy, Rega Institute for Medical Research, Katholieke Universiteit Leuven, Leuven, Belgium

Corresponding author: Tel: +32 16337 388; Fax: +32 16337 387;

E-mail: Arthur.VanAerschot@REGA.KULeuven.ac.be

Current standard therapy for the treatment of chronic infections with hepatitis $C$ virus consists of combination therapy with (pegylated) interferon$\alpha$ and ribavirin. 1,5-Anhydrohexitol nucleoside analogues are constrained congeners known to mimic the ribonucleoside conformation. Within this series some analogues are endowed with strong antiviral properties, particularly against herpesviruses. The six-membered anhydrohexitol ring was, therefore, combined with the triazolyl carboxamide moiety of ribavirin, thus providing a new series of ribavirin analogues. None of the newly synthesized compounds elicited any substantial antiviral activity, neither against herpes simplex virus type 1 and 2, nor against bovine viral diarrhoea virus (a surrogate for hepatitis C virus) or the yellow fever virus.

Keywords: synthesis, antiviral, ribavirin, anhydrohexitol nucleosides, HCV

\section{Introduction}

Worldwide, more than 170 million people are chronic carriers of the hepatitis $\mathrm{C}$ virus $(\mathrm{HCV})$ and are at high risk of developing fatal liver disease. The current treatment is based on a combination therapy of (pegylated) interferon- $\alpha$ and ribavirin (Barnard, 2001; Scot \& Perry, 2002; Wilkinson, 2001). It may be of interest to design more potent analogues of ribavirin that can be used in the combination therapy with interferon. One such analogue, EICAR (5-ethynyl-1$\beta$-D-ribofuranosylimidazole-4-carboxamide) (Balzarini et al., 1993; De Clercq et al., 1991; Neyts et al., 1996), is 30to 50 -fold more potent than ribavirin against various RNA viruses, including flaviviruses.

Several possible mechanisms have been reported by which ribavirin may exert antiviral activity. The 5'monophosphate derivative of ribavirin is a potent inhibitor of IMP dehydrogenase (Sidwell et al., 1979), thus leading to decreased intracellular GTP pools and as a consequence reduced viral replication. Although IMP dehydrogenase is a cellular enzyme, it can be envisaged as a suitable target for antiviral intervention because of the increased need for DNA and RNA building blocks in the virus-infected cells. Ribavirin (as is $5^{\prime}$-monophosphate derivative) is a well-known inhibitor of this enzyme; it is approved for the treatment of respiratory syncytial virus infections and HCV infections. EICAR, as its monophosphate form, has been shown to be a more potent inhibitor of IMP dehydrogenase than ribavirin and this correlates with a more potent antiviral activity (Balzarini et al., 1993; De Clercq et al., 1991).
The $5^{\prime}$-triphosphate metabolite of ribavirin may in addition inhibit the guanylyltransferase and viral RNAdependent RNA polymerase ( $\mathrm{RdRp}$ ), thus leading to, respectively, (i) inhibition of viral CAP formation (at least for those viruses that acquire a CAP via the action of the guanylyltransferase); and (ii) inhibition of $\mathrm{RdRp}$ activity. More recently, ribavirin was also reported to be able to induce an 'error-catastrophy', whereby ribavirin-induced mutagenesis leads to less viable, or unviable, virus (Crotty et al., 2002; Crotty et al., 2000).

1,5-Anhydrohexitol nucleoside analogues are constrained analogues known to mimic the ribonucleoside conformation. Some of these compounds (for example, the 5-iodouracil, 5-ethyluracil, cytosine and guanine base-containing congeners, Figure 1) have previously been reported as selective inhibitors of viral replication (Verheggen et al., 1993; Verheggen et al., 1995). Combination of the sixmembered hexitol moiety with the triazole carboxamide heterocycle, therefore, was a logical extension to the series (Figure 2).

\section{Materials and methods}

\section{General methods}

The ${ }^{1} \mathrm{H}$ and ${ }^{13} \mathrm{C}$ NMR spectra were recorded in the solvent indicated with a Varian Gemini 200 spectrometer and for some cases a Varian 500 Unity spectrometer at $300 \mathrm{~K}$, unless otherwise indicated. For ${ }^{1} \mathrm{H}$ spectra taken in $\mathrm{CDCl}_{3}$ tetramethylsilane (TMS) was used as reference. For spectra 
Figure 1. Chemical structure of the known antiviral agents ribavirin and EICAR, and of the most relevant anhydrohexitol nucleoside analogues (Verheggen et al., 1995)
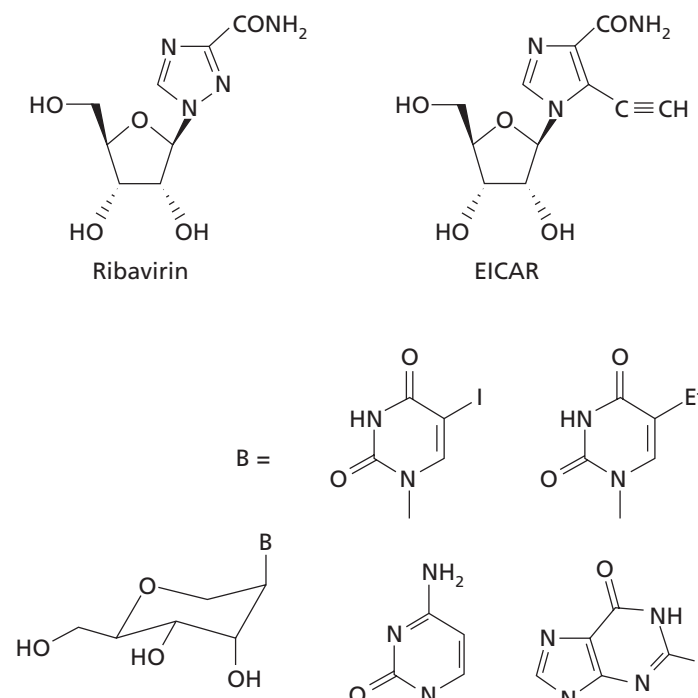<smiles>Cn1cc(I)c(=O)[nH]c1=O</smiles>
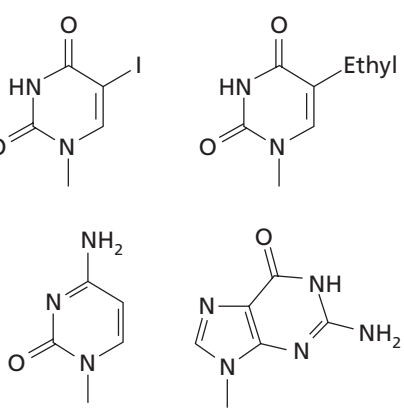

1,5-Anhydrohexitol analogues

taken in DMSO- $\mathrm{d}_{6}$, the solvent signal at $2.5 \mathrm{ppm}$ was used as reference. For the ${ }^{13} \mathrm{C}$ spectra, the solvent peaks were used as reference (for example, $77.00 \mathrm{ppm}$ for $\mathrm{CDCl}_{3}$ and $39.6 \mathrm{ppm}$ for DMSO- $\mathrm{d}_{6}$ ). Exact mass measurements were performed on a quadrupole/orthogonal-acceleration timeof-flight (Q/oaTOF) tandem mass spectrometer (qTof 2, Micromass, Manchester, UK) equipped with a standard electrospray ionization (ESI) interface. Samples were infused in a 2 -propanol/water (1:1) mixture at $3 \mu \mathrm{l} / \mathrm{min}$. Precoated Machery-Nagel Alugram ${ }^{\circledR}$ SilG/UV 254 plates were used for TLC and spots were examined with ultraviolet light and sulfuric acid/anisaldehyde spray. Column chromatography was performed on ICN silica gel (63-200 nm, $60 \AA ̊$ ). Anhydrous solvents were obtained storing analytical grade solvents on $3 \AA$ A molecular sieves for 3 days. Methyl 1H-1,2,4-triazole-3-carboxylate was obtained from Alkemi (Belgium); 4,6-O-benzylidene-1,5-anhydrohexitol was from CMS-chemicals (UK) and methyl glucopyranoside was bought from Acros Organics (Belgium).

Purity of all compounds obtained was verified by TLC analysis, and identity was verified by $\mathrm{MS},{ }^{1} \mathrm{H}$ and ${ }^{13} \mathrm{C}$ NMR analysis. In view of the lack of meaningful antiviral activity, no elementary analysis was carried out.

\section{Antiviral evaluation}

Anti-bovine viral diarrhoea virus (BVDV, PE515 strain) activity was assessed on MDBK cells and anti-yellow fever virus (YFV, vaccine strain 17D) activity on Vero cells. Briefly, confluent cultures grown in 96 plates were infected with $100 \mathrm{CCID}_{50} /$ well of virus and cultures were further incubated at $37^{\circ} \mathrm{C}$ for another 5 (BVDV) or 7 days (YFV), after which antiviral activity was recorded microscopically. Evaluation of activity against vesicular stomatitis virus, parainfluenza- 3 virus, reovirus- 1 , Sindbis virus, Coxsackie virus $\mathrm{B} 4$, Punta Toro virus, respiratory syncytial virus, herpes simplex virus type 1 and 2, vaccinia virus was performed as described earlier (Neyts et al., 1995).

\section{Synthesis of the 1,5-anhydrohexitol nucleoside analogues}

1,5-Anhydro-4,6-O-benzylidene-2-(methyl 1H-1,2,4triazol-1-yl-3-carboxylate)-2-deoxy-D-altro-hexitol (6)

1,2,4-Triazole-3-methylcarboxylate $(1.27 \mathrm{~g}, 10 \mathrm{mmol})$ was suspended in $50 \mathrm{ml}$ of anhydrous DMF, to which was added under argon $380 \mathrm{mg}$ of a $60 \%$ oil dispersion of sodium hydride $(9.5 \mathrm{mmol})$ and the mixture was heated on an oil bath for $30 \mathrm{~min}$ at $90^{\circ} \mathrm{C}$. The allitol epoxide 5 (Allart et al., 1999) (703 mg, $3 \mathrm{mmol}$ ) was added and the mixture was heated for $15 \mathrm{~h}$ at $90^{\circ} \mathrm{C}$, after which the reaction was cooled, quenched with sodium bicarbonate and concentrated. The residue was partitioned between $200 \mathrm{ml}$ of ethyl acetate and $200 \mathrm{ml}$ of $5 \%$ aqueous sodium bicarbonate, and the organics were washed twice with brine. Purification of the organic residue on $70 \mathrm{~g}$ of silica gel (slow gradient of $\mathrm{CH}_{3} \mathrm{OH}$ in $\mathrm{CH}_{2} \mathrm{Cl}_{2}$ 0-2\%) afforded $560 \mathrm{mg}(1.55 \mathrm{mmol}$, $52 \%)$ of the title compound $\mathbf{6}$ as a foam.

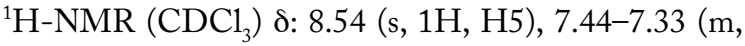
$5 \mathrm{H}$, arom-H), 5.53 (s, $1 \mathrm{H}, \mathrm{PhCH}), 4.62\left(\mathrm{~m}, 2 \mathrm{H}, \mathrm{H} 2^{\prime}\right.$, $\mathrm{H} 3^{\prime}$ ), 4.42 (dd, $1 \mathrm{H}, \mathrm{J}=2.4$ and $\left.13.6 \mathrm{~Hz}, \mathrm{H} 1^{\prime} \mathrm{e}\right), 4.37$ (dd, $1 \mathrm{H}, \mathrm{J}=4.9$ and $\left.10.2 \mathrm{~Hz}, \mathrm{H}^{\prime} \mathrm{e}\right), 4.26(\mathrm{~d}, 1 \mathrm{H}, \mathrm{J}=13.2 \mathrm{~Hz}$, H1'a), 4.08 (dt, 1H, J=4.9 and $10.0 \mathrm{~Hz}, \mathrm{H} 5^{\prime}$ ), 4.02 (s, 3H, $\mathrm{COOCH}_{3}$ ), 3.71 (t, 1H, J=10.2 Hz, H6'a), 3.52 (dd, 1H, $\mathrm{J}=2.4$ and $9.8 \mathrm{~Hz}, \mathrm{H} 4^{\prime}$ ) ppm;

${ }^{13} \mathrm{C}-\mathrm{NMR}\left(\mathrm{CDCl}_{3}\right)$ \&: 159.96 (CO), 154.86 (C3), 144.40 (C5), 136.75 (Ci), 129.33 (Cp), 128.31 (Co), $126.06(\mathrm{Cm}), 102.16(\mathrm{PhCH}), 76.18\left(\mathrm{C}^{\prime}\right), 68.78\left(\mathrm{C}^{\prime}\right)$, $67.31\left(\mathrm{C}^{\prime}\right), 66.92\left(\mathrm{C} 3^{\prime}\right), 64.32\left(\mathrm{C} 1^{\prime}\right), 61.13\left(\mathrm{C}^{\prime}\right), 52.83$ $\left(\mathrm{OCH}_{3}\right)$;

ESI-MS pos.: calcd. for $\mathrm{C}_{17} \mathrm{H}_{20} \mathrm{~N}_{3} \mathrm{O}_{6} 362.1352$; found $362.1370[\mathrm{M}+\mathrm{H}]^{+}$.

1,5-Anhydro-2-(methyl 1H-1,2,4-triazol-1-yl-3-carboxylate)-2-deoxy-D-altro-hexitol (8)

The triazole methylcarboxylate analogue $6(400 \mathrm{mg}, 1.1$ $\mathrm{mmol}$ ) obtained in the previous preparation was dissolved in $20 \mathrm{ml}$ of methanol and $1 \mathrm{ml}$ of TFA was added. The mixture was stirred for $3 \mathrm{~h}$, after which the mixture was evaporated and co-evaporated with dioxane. The title product partially crystallized from a methanol/toluene 
mixture affording $144 \mathrm{mg}(0.52 \mathrm{mmol}, 48 \%)$ of the title compound.

${ }^{1} \mathrm{H}-\mathrm{NMR}$ (DMSO) $\delta: 8.76$ (s, 1H, H5), 5.40 (d, 1H, $\left.\mathrm{J}=4.4 \mathrm{~Hz}, 4^{\prime}-\mathrm{OH}\right), 4.80\left(\mathrm{~d}, 1 \mathrm{H}, \mathrm{J}=6.3 \mathrm{~Hz}, 3^{\prime}-\mathrm{OH}\right), 4.56$ (t, $\left.1 \mathrm{H}, \mathrm{J}=5.6 \mathrm{~Hz}, 6^{\prime}-\mathrm{OH}\right), 4.50$ (dt, 1H, H2'), 4.14 (dd, 1H, $\left.\mathrm{H} 3^{\prime}\right), 4.12\left(\mathrm{dd}, 1 \mathrm{H}, \mathrm{J}=3.0\right.$ and $12.7 \mathrm{~Hz}, \mathrm{H} 1_{\mathrm{e}}{ }^{\prime}$ ), 4.01 (dd, $1 \mathrm{H}, \mathrm{J}=3.4$ and $12.7 \mathrm{~Hz}, \mathrm{H}^{\prime}{ }_{\mathrm{a}}$ ), 3.64-3.57 (m, 2H, H5', H6'A), 3.52 (dd, $\mathrm{J}_{5^{\prime}, 6 \mathrm{~B}}=4.9$ and $\left.\mathrm{J}_{6^{\prime}, 6^{\prime}}=11.2 \mathrm{~Hz}, 1 \mathrm{H}, \mathrm{H} 6^{\prime} \mathrm{B}\right)$, 3.44-3.39 (m, 1H, H4'), 3.30 (s, 3H, $\left.\mathrm{OCH}_{3}\right)$;

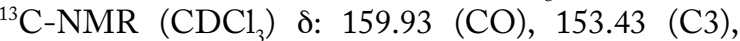
145.55 (C5), $77.84\left(\mathrm{C}^{\prime}\right), 67.87\left(\mathrm{C} 3^{\prime}\right), 64.49\left(\mathrm{C}^{\prime}\right), 62.79$ $\left(\mathrm{C} 1^{\prime}\right), 60.97\left(\mathrm{C} 2^{\prime}\right), 60.39\left(\mathrm{C} 6^{\prime}\right), 52.14\left(\mathrm{OCH}_{3}\right)$;

ESI-MS pos.: calcd. for $\mathrm{C}_{10} \mathrm{H}_{16} \mathrm{~N}_{3} \mathrm{O}_{6}$ 274.1039; found $274.1048[\mathrm{M}+\mathrm{H}]^{+}$.

\section{1,5-Anhydro-4,6-O-benzylidene-2-(1H-1,2,4-triazol-1-} yl-3-carboxamide)-2-deoxy-D-altro-hexitol (9)

The methyl triazolylcarboxylate derivative $6(530 \mathrm{mg}, 1.47$ $\mathrm{mmol}$ ) was dissolved in $30 \mathrm{ml}$ of a $2 \mathrm{M}$ solution of ammonia in methanol and stirred overnight at room temperature, after which the mixture was evaporated and the residue was adsorbed on silica gel. Chromatographic purification (0-5\% gradient of $\mathrm{CH}_{3} \mathrm{OH}$ in $\mathrm{CH}_{2} \mathrm{Cl}_{2}$ ) yielded $380 \mathrm{mg}$ $(1.1 \mathrm{mmol}, 75 \%)$ of $\mathbf{9}$ as a foam.

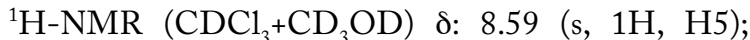

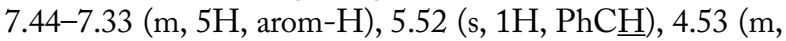
2H, H2', H3'), 4.39 (dd, $1 \mathrm{H}, \mathrm{J}=1.7$ and $\left.13.5 \mathrm{~Hz}, \mathrm{H} 1^{\prime} \mathrm{e}\right), 4.30$ (d, $\left.1 \mathrm{H}, \mathrm{J}=13.7 \mathrm{~Hz}, \mathrm{H}^{\prime} \mathrm{a}\right), 4.29$ (dd, $1 \mathrm{H}, \mathrm{J}=5.3$ and $10.2 \mathrm{~Hz}$, H6'e), 4.08 (dt, 1H, J=4.9 and $\left.10.0 \mathrm{~Hz}, \mathrm{H} 5^{\prime}\right), 3.70$ (t, 1H, $\mathrm{J}=10.5 \mathrm{~Hz}, \mathrm{H}^{\prime} \mathrm{a}$ ), 3.55 (dd, $1 \mathrm{H}, \mathrm{J}=2.0$ and $9.8 \mathrm{~Hz}, \mathrm{H} 4^{\prime}$ );

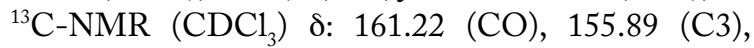
143.94 (C5), 136.75 (Ci), 128.35 (Cp), 127.40 (Co), $125.61(\mathrm{Cm}), 101.61$ (Ph్H), $75.85\left(\mathrm{C}^{\prime}\right), 68.16$ (C6'), $66.36\left(\mathrm{C}^{\prime}\right), 65.82\left(\mathrm{C} 3^{\prime}\right), 63.47\left(\mathrm{C}^{\prime}\right), 61.52\left(\mathrm{C} 2^{\prime}\right)$;

ESI-MS pos.: calcd. for $\mathrm{C}_{16} \mathrm{H}_{19} \mathrm{~N}_{4} \mathrm{O}_{5} 347.1355$; found $347.1328[\mathrm{M}+\mathrm{H}]^{+}$.

\section{1,5-Anhydro-2-(1H-1,2,4-triazol-1-yl-3-carboxamide)- 2-deoxy-D-altro-hexitol (1)}

An amount of $380 \mathrm{mg}(1.1 \mathrm{mmol})$ of the triazole carboxamide analogue 9 was dissolved in $20 \mathrm{ml}$ of methanol and $1 \mathrm{ml}$ of TFA was added. The mixture was stirred for $4 \mathrm{~h}$, after which a precipitate was formed. After evaporation and co-evaporation with dioxane the mixture was crystallized from boiling ethanol affording $223 \mathrm{mg}$ (0.86 mmol, 78\%) of the title compound $\mathbf{1}$.

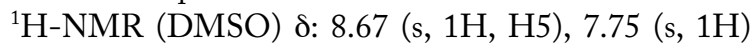
and $7.54(\mathrm{~s}, 1 \mathrm{H})\left(\mathrm{NH}_{2}\right), 5.40\left(\mathrm{~d}, 1 \mathrm{H}, \mathrm{J}=4.4 \mathrm{~Hz}, 3^{\prime}-\mathrm{OH}\right)$, 4.78 (d, 1H, J=5.8 Hz, 4'-OH), 4.55 (t, 1H, 6'-OH), 4.45 $\left(\mathrm{dt}, 1 \mathrm{H}, \mathrm{J}_{2^{\prime}, 3^{\prime}}=5.1\right.$ and $\left.\mathrm{J}_{2^{\prime}, 1^{\prime}}=3.2 \mathrm{~Hz}, \mathrm{H} 2^{\prime}\right), 4.14(\mathrm{dd}, 1 \mathrm{H}, \mathrm{J}=3.2$ and $\left.4.9 \mathrm{~Hz}, \mathrm{H} 3^{\prime}\right), 4.12$ (dd, $1 \mathrm{H}, \mathrm{J}=3.0$ and $12.5 \mathrm{~Hz}, \mathrm{H} 1_{\mathrm{e}}^{\prime}$ ), $4.01\left(\mathrm{dd}, 1 \mathrm{H}, \mathrm{J}=3.2\right.$ and $\left.12.5 \mathrm{~Hz}, \mathrm{H} 1_{\mathrm{a}}^{\prime}\right), 3.63\left(\mathrm{dd}, \mathrm{J}_{5^{\prime}, 6^{\prime} \mathrm{A}}=2.9\right.$ and $\mathrm{J}_{6^{\prime}, 6^{\prime}}=11.3 \mathrm{~Hz}, 1 \mathrm{H}, \mathrm{H} 6^{\prime} \mathrm{A}$ ), 3.60 (ddd, $\mathrm{J}=2.9,5.0$ and 7.9
Figure 2. Main target nucleoside analogues 1-4, resulting from combination of the 1,5-anhydrohexitol moiety with the heterocyclic base 1,2,4-triazole3-carboxamide as in ribavirin

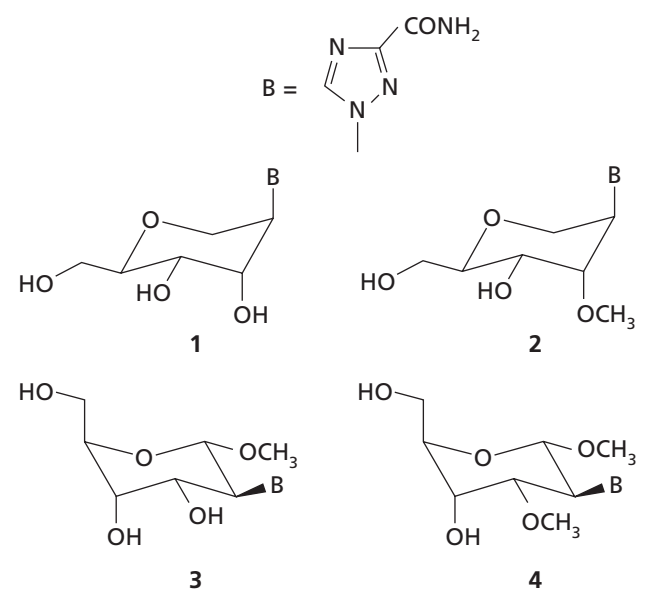

Structures $\mathbf{3}$ and $\mathbf{4}$ have a different conformation due to the steric repulsion between the substituents on carbons 1' and 3'.

$\left.\mathrm{Hz}, 1 \mathrm{H}, \mathrm{H} 5^{\prime}\right), 3.52\left(\mathrm{dd}, \mathrm{J}_{5^{\prime}, 6^{\prime} \mathrm{B}}=5.0\right.$ and $\mathrm{J}_{6^{\prime}, 6^{\prime}}=11.3 \mathrm{~Hz}, 1 \mathrm{H}$, $\mathrm{H} 6^{\prime} \mathrm{B}$ ), 3.38 (dd, $1 \mathrm{H}, \mathrm{J}_{3^{\prime}, 4^{\prime}}=3.2$ and $\mathrm{J}_{4^{\prime}, 5^{\prime}}=7.8 \mathrm{~Hz}, \mathrm{H} 4^{\prime}$ );

${ }^{13} \mathrm{C}-\mathrm{NMR}\left(\mathrm{CDCl}_{3}\right)$ \&: 160.55 (CO), 156.53 (C3), 144.70 (C5), $77.73\left(\mathrm{C5}^{\prime}\right), 67.67$ (C3'), 64.20 (C4'), 62.62 $\left(\mathrm{C}^{\prime}\right), 60.59$ (C2'), $60.21\left(\mathrm{C}^{\prime}\right)$;

ESI-MS pos.: calcd. for $\mathrm{C}_{9} \mathrm{H}_{15} \mathrm{~N}_{4} \mathrm{O}_{5}$ 259.1042; found $259.1048[\mathrm{M}+\mathrm{H}]^{+}$.

\section{1,5-Anhydro-4,6-O-benzylidene-3-O-methyl-2- (methyl 1H-1,2,4-triazol-1-yl-3-carboxylate)-2-deoxy- D-altro-hexitol (7)}

An amount of $900 \mathrm{mg}(2.5 \mathrm{mmol})$ of the triazolide 6 was dissolved under argon in $20 \mathrm{ml}$ dry DMF and cooled on an ice bath. A $60 \% \mathrm{NaH}$ dispersion in oil was added $(120 \mathrm{mg}$, $3 \mathrm{mmol}$ ) and the mixture was stirred for $45 \mathrm{~min}$ at $0^{\circ} \mathrm{C}$, after which methyl iodide $(0.25 \mathrm{ml}, 4 \mathrm{mmol})$ dissolved in $10 \mathrm{ml}$ of $\mathrm{DMF}$ was added over $30 \mathrm{~min}$. After stirring for $3 \mathrm{~h}$ at room temperature, the reaction was quenched with $3 \mathrm{ml}$ of water and stirred further for $10 \mathrm{~min}$. Following concentration, the mixture was diluted with $70 \mathrm{ml}$ of ethyl acetate and washed twice with $50 \mathrm{ml}$ of $5 \%$ aqueous $\mathrm{NaHCO}_{3}$ and once with 50 $\mathrm{ml}$ of brine. The aqueous washes were again extracted with $50 \mathrm{ml}$ of ethyl acetate. Chromatographic purification on silica gel $\left(\mathrm{CH}_{2} \mathrm{Cl}_{2}\right.$ to $\mathrm{CH}_{2} \mathrm{Cl}_{2} / \mathrm{CH}_{3} \mathrm{OH}$ 98:2) afforded $647 \mathrm{mg}$ (1.72 mmol, 70\%) of the sugar methylated product 7 .

${ }^{1} \mathrm{H}-\mathrm{NMR}\left(\mathrm{CDCl}_{3}\right)$ \&: 8.57 (s, 1H, H5), 7.45-7.33 (m, $5 \mathrm{H}$, arom-H), 5.40 (s, $1 \mathrm{H}, \mathrm{PhCH}), 4.61(\mathrm{t}, 1 \mathrm{H}, \mathrm{J}=2.4 \mathrm{~Hz}$, $\mathrm{H} 2^{\prime}$ ), 4.38 (dd, 1H, J=2.4 and $13.2 \mathrm{~Hz}, \mathrm{H} 1^{\prime} \mathrm{e}$ ), 4.34 (dd, $1 \mathrm{H}, \mathrm{J}=5.4$ and $\left.10.2 \mathrm{~Hz}, \mathrm{H}^{\prime} \mathrm{e}\right), 4.31$ (d, 1H, J=13.2 Hz, $\left.\mathrm{H} 1^{\prime} \mathrm{a}\right), 4.23$ (t, 1H, J=5.9 Hz, H3'), 4.11 (dt, 1H, J=5.1 and $\left.9.9 \mathrm{~Hz}, \mathrm{H}^{\prime}\right), 4.03$ (s, 3H, $\mathrm{COOCH}_{3}$ ), 3.66 (s, 3H, 3'- 
$\mathrm{OCH}_{3}$ ), 3.65 (t, 1H, J=10.5 Hz, H6'a), 3.44 (dd, 1H, J=2.7 and $\left.9.5 \mathrm{~Hz}, \mathrm{H} 4^{\prime}\right)$;

${ }^{13} \mathrm{C}-\mathrm{NMR}\left(\mathrm{CDCl}_{3}\right)$ 8: $160.02(\mathrm{CO}), 155.00(\mathrm{C} 3), 144.40$ (C5), 137.20 (Ci), $129.10(\mathrm{Cp}), 128.24(\mathrm{Co}), 126.09(\mathrm{Cm})$, $102.42(\mathrm{Ph} \underline{\mathrm{CH}}), 75.54\left(\mathrm{C}^{\prime}\right), 68.93\left(\mathrm{C}^{\prime}\right), 67.55\left(\mathrm{C} 5^{\prime}\right)$, $64.16\left(\mathrm{C}^{\prime}\right), 60.76$ and $60.23\left(\mathrm{C}^{\prime}, \mathrm{C} 2^{\prime}\right), 52.79\left(2 \mathrm{xOCH}_{3}\right)$;

ESI-MS pos.: calcd. for $\mathrm{C}_{18} \mathrm{H}_{22} \mathrm{~N}_{3} \mathrm{O}_{6} 376.1509$; found $376.1496[\mathrm{M}+\mathrm{H}]^{+}$.

\section{1,5-Anhydro-3-O-methyl-2-(1H-1,2,4-triazol-1-yl-3- carboxamide)-2-deoxy-D-altro-hexitol (2)}

The foam 7 obtained in the previous preparation $(408 \mathrm{mg}$, $1.08 \mathrm{mmol}$ ) was dissolved in $25 \mathrm{ml}$ of a $2 \mathrm{M}$ solution of ammonia in methanol and stirred overnight at room temperature, after which the mixture was evaporated and the residue was adsorbed on silica gel. Purification on silica gel (gradient of $\mathrm{CH}_{3} \mathrm{OH}$ in $\mathrm{CH}_{2} \mathrm{Cl}_{2} 0-10 \%$ ) yielded $350 \mathrm{mg}$ (0.97 mmol, $90 \%)$ of $\mathbf{1 0}$ as a white foam.

ESI-MS pos. calcd. for $\mathrm{C}_{17} \mathrm{H}_{21} \mathrm{~N}_{4} \mathrm{O}_{5} 361.1512$; found $361.1534[\mathrm{M}+\mathrm{H}]^{+}$.

Following dissolution of the foam in $25 \mathrm{ml}$ of methanol, $1.25 \mathrm{ml}$ of TFA were added and the mixture was stirred for $4 \mathrm{~h}$ at room temperature. Evaporation left an oil that was crystallized from methanol/diisopropyl ether, affording 188 $\mathrm{mg}(0.69 \mathrm{mmol}, 71 \%)$ of the title compound 2.

${ }^{1} \mathrm{H}-\mathrm{NMR}\left(\mathrm{DMSO}-\mathrm{d}_{6}\right)$ 8: $8.70(\mathrm{~s}, 1 \mathrm{H}, \mathrm{H} 5), 7.77(\mathrm{~s}, 1 \mathrm{H})$ and $7.56(\mathrm{~s}, 1 \mathrm{H})\left(\mathrm{NH}_{2}\right), 5.0-4.6(\mathrm{br}, 2 \mathrm{xOH}), 4.66(\mathrm{dt}, 1 \mathrm{H}$, $\mathrm{J}_{2^{\prime}, 3^{\prime}}=4.9$ and $\left.\mathrm{J}_{2^{\prime}, 1^{\prime}}=3.5 \mathrm{~Hz}, \mathrm{H} 2^{\prime}\right), 4.15(\mathrm{dd}, 1 \mathrm{H}, \mathrm{J}=3.6$ and $12.5 \mathrm{~Hz}, \mathrm{H} 1_{\mathrm{e}}^{\prime}$ ), 3.94 (dd, $1 \mathrm{H}, \mathrm{J}=3.9$ and $12.7 \mathrm{~Hz}, \mathrm{H}^{\prime}{ }_{\mathrm{a}}$ ), $3.87\left(\mathrm{dd}, 1 \mathrm{H}, \mathrm{J}=2.9\right.$ and $\left.4.9 \mathrm{~Hz}, \mathrm{H} 3^{\prime}\right), 3.62\left(\mathrm{dd}, \mathrm{J}_{5^{\prime}, 6^{\prime} \mathrm{A}}=2.9\right.$ and $\left.\mathrm{J}_{6^{\prime}, 6^{\prime}}=11.3 \mathrm{~Hz}, 1 \mathrm{H}, \mathrm{H} 6^{\prime} \mathrm{A}\right), 3.60-3.54\left(\mathrm{~m}, 2 \mathrm{H}, \mathrm{H} 4^{\prime}\right.$, $\left.\mathrm{H} 5^{\prime}\right), 3.52\left(\mathrm{dd}, \mathrm{J}_{5^{\prime}, 6^{\prime} \mathrm{B}}=4.7\right.$ and $\mathrm{J}_{6^{\prime}, 6^{\prime}}=11.0 \mathrm{~Hz}, 1 \mathrm{H}, \mathrm{H}^{\prime} \mathrm{B}$ ), $3.42\left(\mathrm{~s}, 3 \mathrm{H}, \mathrm{OCH}_{3}\right)$;

${ }^{13} \mathrm{C}-\mathrm{NMR}\left(\mathrm{CDCl}_{3}\right)$ 8: $160.53(\mathrm{CO}), 156.65$ (C3), 144.93 (C5), 78.38, 77.91 (C3', C5'), 63.38, 63.12 (C4', $\left.\mathrm{C} 1^{\prime}\right), 60.21,58.08,57.80\left(\mathrm{C2}^{\prime}, \mathrm{C}^{\prime}, \mathrm{OCH}_{3}\right)$;

ESI-MS pos.: HRMS calcd. for $\mathrm{C}_{10} \mathrm{H}_{17} \mathrm{~N}_{4} \mathrm{O}_{5}$ 273.1199; found $273.1189[\mathrm{M}+\mathrm{H}]^{+}$.

\section{Synthesis of the methyl pyranoside nucleoside analogues}

\section{Methyl 1,5;2,3-dianhydro-4,6-O-benzylidene-D-allo- hexopyranoside (11)}

The title compound was prepared largely following literature procedures (Richtmeyer \& Hudson, 1941; Rosenfeld et al., 1948).

${ }^{1} \mathrm{H}-\mathrm{NMR}\left(\mathrm{CDCl}_{3}\right)$ 8: 7.52-7.33 (m, 5H, arom-H), 5.56 (s, $1 \mathrm{H}, \mathrm{PhC} \underline{\mathrm{H}}), 4.88(\mathrm{~d}, 1 \mathrm{H}, \mathrm{J}=2.7 \mathrm{~Hz}, \mathrm{H} 1), 4.24(\mathrm{dd}, 1 \mathrm{H}$, $\mathrm{J}=5.0$ and $\left.10.2 \mathrm{~Hz}, \mathrm{H6}^{\prime} \mathrm{e}\right), 4.08$ (m, 1H, H5), 3.94 (dd, 1H, $\mathrm{J}=1.1$ and $9.1 \mathrm{~Hz}, \mathrm{H} 4), 3.68\left(\mathrm{t}, 1 \mathrm{H}, \mathrm{J}=6.9 \mathrm{~Hz}, \mathrm{H} 6{ }^{\prime} \mathrm{a}\right), 3.51$ $(\mathrm{dm}, 1 \mathrm{H}, \mathrm{J}=4.4 \mathrm{~Hz}, \mathrm{H} 3), 3.48(\mathrm{dd}, 1 \mathrm{H}, \mathrm{J}=2.9$ and $4.4 \mathrm{~Hz}$, $\mathrm{H} 2), 3.47$ (s, 3H, $\mathrm{OCH}_{3}$ );
${ }^{13} \mathrm{C}-\mathrm{NMR}\left(\mathrm{CDCl}_{3}\right)$ 8: $137.18(\mathrm{Ci}), 129.20(\mathrm{Cp}), 128.29$ (Cm), 126.32 (Co), 102.77 (Ph్ㄱ), 95.35 (C1), 77.92 (C4), $68.92(\mathrm{C} 6), 60.06(\mathrm{C} 5), 55.82\left(\mathrm{OCH}_{3}\right), 53.10(\mathrm{C} 2)$, $50.68(\mathrm{C} 3)$;

ESI-MS pos.: calcd. for $\mathrm{C}_{14} \mathrm{H}_{17} \mathrm{O}_{5}$ 265.1076; found $265.1082[\mathrm{M}+\mathrm{H}]^{+}$.

\section{Methyl 4,6-O-benzylidene-2-(methyl 1H-1,2,4- triazol-1-yl-3-carboxylate)-2-deoxy-D-altro- hexopyranoside (12)}

Methyl 1H-1,2,4-triazole-3-carboxylate $(5.72 \mathrm{~g}, 45 \mathrm{mmol})$ was suspended in $100 \mathrm{ml}$ of anhydrous DMF, to which was added under argon $1.72 \mathrm{~g}$ of a $60 \%$ oil dispersion of sodium hydride $(43 \mathrm{mmol})$ and the mixture was heated on an oil bath for $1 \mathrm{~h}$ at $90^{\circ} \mathrm{C}$. The alloside epoxide $\mathbf{1 1}(3.52 \mathrm{~g}, 15$ $\mathrm{mmol}$ ) was added and the mixture was heated for $48 \mathrm{~h}$ at $90^{\circ} \mathrm{C}$, after which TLC analysis indicated the formation of several minor products. The reaction was cooled, quenched with sodium bicarbonate and concentrated. The residue was partitioned between $200 \mathrm{ml}$ of ethyl acetate and $200 \mathrm{ml}$ of $5 \%$ aqueous sodium bicarbonate, the aqueous phase was extracted twice more and the organics were washed twice with brine. Purification of the organic residue on $70 \mathrm{~g}$ of silica gel (slow gradient of $\mathrm{CH}_{3} \mathrm{OH}$ in $\mathrm{CH}_{2} \mathrm{Cl}_{2}$ 0-2\%) afforded $1220 \mathrm{mg}$ (3.12 mmol, 21\%) of the title compound $\mathbf{1 2}$ as a foam, which was crystallized from acetone/diisopropyl ether.

${ }^{1} \mathrm{H}-\mathrm{NMR}\left(\mathrm{CDCl}_{3}\right)$ 8: 8.40 (s, 1H, H5), 7.46-7.34 (m, $5 \mathrm{H}$, arom-H), 5.62 (s, 1H, $\mathrm{PhC} \underline{\mathrm{H}}), 5.05\left(\mathrm{~s}, 1 \mathrm{H}, \mathrm{H} 1^{\prime}\right), 4.87$ (d, $\left.1 \mathrm{H}, \mathrm{J}=2.4 \mathrm{~Hz}, \mathrm{H} 2^{\prime}\right), 4.45-4.39$ (m, 3H, H3', H5', $\left.\mathrm{H}^{\prime} \mathrm{e}\right), 4.01\left(\mathrm{~s}, 3 \mathrm{H}, \mathrm{COOCH}_{3}\right), 3.89$ (dd, J=3.4 and $9.3 \mathrm{~Hz}$, 1H, H4'), 3.87 (t, J=11.7 Hz, 1H, H6'a), 3.52 (s, 3H, 1'$\mathrm{OCH}_{3}$;

${ }^{13} \mathrm{C}-\mathrm{NMR}\left(\mathrm{CDCl}_{3}\right)$ 8: 159.81 (CO), 155.11 (C3), $144.20(\mathrm{C} 5), 136.78(\mathrm{Ci}), 129.31(\mathrm{Cp}), 128.29(\mathrm{Cm})$, 126.15 (Co), $102.25(\mathrm{Ph} \underline{\mathrm{CH}}), 98.95\left(\mathrm{C1}^{\prime}\right), 75.26\left(\mathrm{C}^{\prime}\right)$, $68.92\left(\mathrm{C} 6^{\prime}\right), 67.75\left(\mathrm{C}^{\prime}\right), 62.88\left(\mathrm{C}^{\prime}\right), 58.31\left(\mathrm{C}^{\prime}\right), 56.03$ $\left(1^{\prime}-\mathrm{OCH}_{3}\right), 52.82\left(\mathrm{COOCH} \underline{\mathrm{H}}_{3}\right)$;

ESI-MS pos. calcd. for $\mathrm{C}_{18} \mathrm{H}_{22} \mathrm{~N}_{3} \mathrm{O}_{7} 392.1458$; found $392.1465[\mathrm{M}+\mathrm{H}]^{+}$.

In addition $800 \mathrm{mg}$ of the epoxide $\mathbf{1 1}(3.0 \mathrm{mmol}, 20 \%)$ was recovered, and $291 \mathrm{mg}(0.87 \mathrm{mmol}, 6 \%)$ of the decarboxylated triazolide $\mathbf{1 3}$ was isolated as a foam.

Methyl 4,6-O-benzylidene-2-(1H-1,2,4-triazol-1-yl)-2deoxy-D-altro-hexopyranoside (13)

${ }^{1} \mathrm{H}-\mathrm{NMR}\left(\mathrm{CDCl}_{3}\right)$ 8: 8.22 (s, 1H, H5), 7.98 (s, 1H, H3), 7.46-7.34 (m, 5H, arom-H), $5.63(\mathrm{~s}, 1 \mathrm{H}, \mathrm{PhCH}), 4.93$ (s, 1H, H1'), 4.75 (d, 1H, J=2.4 Hz, H2'), 4.45-4.38 (m, 2H, H5', H6'e), 4.31 (brs, $1 \mathrm{H}, \mathrm{H}^{\prime}$ ), 4.03 (dd, $1 \mathrm{H}, \mathrm{J}=3$ and $9 \mathrm{~Hz}$, H4'), 3.87 (t, 1H, J=12 Hz, H6'a), 3.49 (s, 3H, 1'- $\mathrm{OCH}_{3}$ );

${ }^{13} \mathrm{C}-\mathrm{NMR}\left(\mathrm{CDCl}_{3}\right)$ 8: 152.13 (C3), 142.97 (C5), $136.96(\mathrm{Ci}), 129.26(\mathrm{Cp}), 128.26(\mathrm{Cm}), 126.17(\mathrm{Co})$, 
$102.19(\mathrm{PhC} H), 99.44\left(\mathrm{C}^{\prime}\right), 75.51\left(\mathrm{C}^{\prime}\right), 69.02\left(\mathrm{C}^{\prime}\right)$, $68.04\left(\mathrm{C}^{\prime}\right), 62.00\left(\mathrm{C}^{\prime}\right), 58.12\left(\mathrm{C}^{\prime}\right), 55.86\left(1^{\prime}-\mathrm{OCH}_{3}\right)$;

ESI-MS pos.: calcd. for $\mathrm{C}_{16} \mathrm{H}_{20} \mathrm{~N}_{3} \mathrm{O}_{5}$ 334.1403; found $334.1418[\mathrm{M}+\mathrm{H}]^{+}$.

Methyl 4,6-O-benzylidene-2-(1H-1,2,4-triazol-1-yl-3carboxamide)-2-deoxy-D-altro-hexopyranoside (14)

The methyl carboxylate $\mathbf{1 2}$ obtained in the previous preparation ( $500 \mathrm{mg}, 1.28 \mathrm{mmol}$ ) was dissolved in $20 \mathrm{ml}$ of a $2 \mathrm{M}$ solution of ammonia in methanol and stirred overnight at room temperature, after which the mixture was evaporated and the residue was adsorbed on silica gel. Flash purification on silica gel (gradient of $\mathrm{CH}_{3} \mathrm{OH}$ in $\mathrm{CH}_{2} \mathrm{Cl}_{2}$ 0-7\%) yielded $450 \mathrm{mg}$ of $\mathbf{1 4}(1.19 \mathrm{mmol}, 93 \%)$ as a foam.

${ }^{1} \mathrm{H}-\mathrm{NMR}\left(\mathrm{DMSO}-\mathrm{d}_{6}\right)$ $\delta: 8.74$ (s, 1H, H5), 7.46-7.34 (m, 5H, arom-H), 5.73 (s, 1H, $\mathrm{PhCH}$ ), 5.50 (d, 1H, 3'$\mathrm{OH}), 5.00$ (s, 1H, H1'), 4.73 (d, 1H, J=2.5 Hz, H2'), 4.25 (dd, $1 \mathrm{H}, \mathrm{J}=5.1$ and $\left.9.5 \mathrm{~Hz}, \mathrm{H} 6^{\prime} \mathrm{e}\right), 4.20(\mathrm{dd}, 1 \mathrm{H}, \mathrm{J}=5.3$ and $9.9 \mathrm{~Hz}, \mathrm{H} 5^{\prime}$ ), 4.17 (t, 1H, J=2.8 Hz, H3'), 4.01 (dd, J=3.2 and $\left.9.5 \mathrm{~Hz}, 1 \mathrm{H}, \mathrm{H} 4^{\prime}\right), 3.82$ (t, J=9.7 Hz, 1H, H6'a), 3.35 (s, 3H, 1'- $\left.\mathrm{OCH}_{3}\right)$;

${ }^{13} \mathrm{C}-\mathrm{NMR}$ (DMSO-d $)$ ): 160.38 (CO), 156.87 (C3), 145.11 (C5), 137.79 (Ci), $128.79(\mathrm{Cp}), 127.92(\mathrm{Cm})$, 126.39 (Co), 100.95 (PhCH), 98.74 (C1'), 75.02 (C4'), $68.17\left(\mathrm{C} 6^{\prime}\right), 66.47\left(\mathrm{C} 3^{\prime}\right), 63.16\left(\mathrm{C}^{\prime}\right), 57.53\left(\mathrm{C}^{\prime}\right), 54.86$ $\left(1^{\prime}-\mathrm{OCH}_{3}\right)$;

ESI-MS pos.: calcd. for $\mathrm{C}_{17} \mathrm{H}_{21} \mathrm{~N}_{4} \mathrm{O}_{6}$ 377.1461; found $377.1450[\mathrm{M}+\mathrm{H}]^{+}$.

Methyl 2-(1H-1,2,4-triazol-1-yl-3-carboxamide)-2deoxy-D-altro-hexopyranoside (3)

The triazole carboxamide derivative 14 (404 mg, 1.07 mmol) was dissolved in $25 \mathrm{ml}$ of methanol, to which was added $0.25 \mathrm{ml}$ of acetic acid. The solution was degassed by bubbling nitrogen for $10 \mathrm{~min}$, after which $250 \mathrm{mg}$ of $10 \%$ $\mathrm{Pd}$ on carbon was added and the mixture was hydrogenated for $4 \mathrm{~h}$ on a Parr apparatus at 45 psi. The mixture was filtered, the filter was washed with hot ethanol, the volatiles were removed in vacuo and the residue was co-evaporated twice with dioxane. Column purification on silica gel (gradient of $\mathrm{CH}_{3} \mathrm{OH}$ in $\mathrm{CH}_{2} \mathrm{Cl}_{2}$ 4-16\%) and crystallisation from $\mathrm{CH}_{3} \mathrm{OH} /$ toluene afforded the title compound 3 in $78 \%$ yield (242 mg, $0.84 \mathrm{mmol})$.

${ }^{1} \mathrm{H}-\mathrm{NMR}$ (DMSO-d 6 ) $\delta: 8.65$ (s, 1H, H5), 7.73 (s, 1H) and $7.53(\mathrm{~s}, 1 \mathrm{H})\left(\mathrm{NH}_{2}\right), 5.04\left(\mathrm{~d}, 1 \mathrm{H}, \mathrm{J}=3 \mathrm{~Hz}, 4^{\prime}-\mathrm{OH}\right), 4.97$ (d, J=6.8 Hz, 1H, H1'), 4.96-4.88 (m, 2H, 3'-OH, 6'$\mathrm{OH}), 4.36\left(\mathrm{dd}, 1 \mathrm{H}, \mathrm{J}_{2^{\prime}, 1^{\prime}}=6.8 \mathrm{~Hz}, \mathrm{~J}_{2^{\prime}, 3^{\prime}}=10.3 \mathrm{~Hz}, \mathrm{H} 2^{\prime}\right)$, 4.08-4.02 (m, 1H, H3'), 3.86-3.80 (m, 2H, H4', H5'), $3.62\left(\mathrm{~m}, 2 \mathrm{H}, \mathrm{H} 6^{\prime}\right), 3.24$ (s, 3H, 1'- $\left.\mathrm{OCH}_{3}\right)$;

${ }^{13} \mathrm{C}-\mathrm{NMR}\left(\mathrm{DMSO}-\mathrm{d}_{6}\right)$ d: 160.67 (CO), 157.11 (C3), 146.41 (C5), 99.10 (C1'), 77.41 (C5'), 67.62 (C3'), 67.07 (C4'), $62.52\left(\mathrm{C}^{\prime}\right), 60.97\left(\mathrm{C}^{\prime}\right), 55.13\left(\mathrm{OCH}_{3}\right)$;
ESI-MS pos.: calcd. for $\mathrm{C}_{10} \mathrm{H}_{17} \mathrm{~N}_{4} \mathrm{O}_{6}$ 289.1148; found $289.1136[\mathrm{M}+\mathrm{H}]^{+}$.

\section{Methyl 4,6-O-benzylidene-3-O-methyl-2-(1H-1,2,4- triazol-1-yl-3-carboxamide)-2-deoxy-D-altro- hexopyranoside (15)}

An amount of $626 \mathrm{mg}(1.60 \mathrm{mmol})$ of the methyl carboxylate 12 was dissolved under argon in $10 \mathrm{ml}$ dry DMF and cooled on an ice bath. A $60 \% \mathrm{NaH}$ dispersion in oil was added ( $84 \mathrm{mg}, 1.9 \mathrm{mmol}$ ) and the mixture was stirred for $45 \mathrm{~min}$ at $0^{\circ} \mathrm{C}$, after which methyl iodide $(0.16 \mathrm{ml}, 2.6$ $\mathrm{mmol}$ ) dissolved in $10 \mathrm{ml}$ of DMF was added over $60 \mathrm{~min}$. After stirring for $2 \mathrm{~h}$ more at room temperature, the reaction was quenched with $3 \mathrm{ml}$ of aqueous sodium bicarbonate and stirred for an additional $10 \mathrm{~min}$. Following concentration, the mixture was partitioned between $2 \times 70 \mathrm{ml}$ of ethyl acetate and $2 \times 50 \mathrm{ml}$ of $5 \%$ aqueous $\mathrm{NaHCO}_{3}$ and $1 \times 50 \mathrm{ml}$ of brine. Chromatographic purification on silica gel $\left(\mathrm{CH}_{2} \mathrm{Cl}_{2}\right.$ to $\mathrm{CH}_{2} \mathrm{Cl}_{2} / \mathrm{CH}_{3} \mathrm{OH}$ 98:2) afforded $276 \mathrm{mg}$ $(0.68 \mathrm{mmol}, 42 \%)$ of the sugar-methylated product.

ESI-MS pos.: calcd. for $\mathrm{C}_{19} \mathrm{H}_{24} \mathrm{~N}_{3} \mathrm{O}_{7}$ 406.1614; found 406.1612 [M+H] $]^{+}$.

The sugar-methylated analogue thus obtained was suspended in $15 \mathrm{ml}$ of a $2 \mathrm{M}$ solution of ammonia in methanol, to which was added $5 \mathrm{ml}$ of dioxane, and the mixture was stirred overnight at room temperature. Thereafter, the mixture was evaporated and the residue was adsorbed on silica gel. Flash purification on silica gel (gradient of $\mathrm{CH}_{3} \mathrm{OH}$ in $\mathrm{CH}_{2} \mathrm{Cl}_{2}$ 0-4\%) yielded $228 \mathrm{mg}(0.58$ $\mathrm{mmol}, 86 \%$ ) of $\mathbf{1 5}$ as a white foam.

${ }^{1} \mathrm{H}-\mathrm{NMR}\left(\mathrm{DMSO}-\mathrm{d}_{6}\right) \delta: 8.79$ (s, 1H, H5), 7.84 (s, 1H) and $7.63(\mathrm{~s}, 1 \mathrm{H})\left(\mathrm{NH}_{2}\right), 7.44-7.35(\mathrm{~m}, 5 \mathrm{H}$, arom-H), 5.71 (s, $1 \mathrm{H}, \mathrm{PhC} \underline{\mathrm{H}}), 5.00$ (s, 1H, H1'), 4.95 (d, 1H, J=2.0 Hz, $\mathrm{H} 2^{\prime}$ ), 4.27 (dd, 1H, J=5.4 and $10.3 \mathrm{~Hz}, \mathrm{H} 6{ }^{\prime} \mathrm{e}$ ), 4.25 (dd, $\mathrm{J}=3.4$ and $\left.9.7 \mathrm{~Hz}, 1 \mathrm{H}, \mathrm{H} 4^{\prime}\right), 4.19(\mathrm{dt}, 1 \mathrm{H}, \mathrm{J}=5.2$ and 10.0 $\mathrm{Hz}, \mathrm{H}^{\prime}$ ), 3.96 (t, 1H, J=2.4 Hz, H3'), 3.81 (t, J=10.0 Hz, 1H, H6'a), 3.47 (s, 3H, $\mathrm{OCH}_{3}$ ), 3.35 (s, 3H, $\mathrm{OCH}_{3}$ );

${ }^{13} \mathrm{C}-\mathrm{NMR}\left(\mathrm{DMSO}-\mathrm{d}_{6}\right.$ ) $\delta: 160.38$ (CO), 156.89 (C3), 145.53 (C5), 137.80 (Ci), 128.86 (Cp), 128.09 (Cm), 126.19 (Co), $101.00(\mathrm{PhC} H), 98.63$ (C1'), 75.94 (C3'), 75.16 (C4'), 68.19 (C6'), $59.55\left(\mathrm{C}^{\prime}\right), 58.69\left(3^{\prime}-\mathrm{OCH}_{3}\right)$, $58.09\left(\mathrm{C}^{\prime}\right), 54.94\left(1^{\prime}-\mathrm{OCH}_{3}\right)$;

ESI-MS pos.: calcd. for $\mathrm{C}_{18} \mathrm{H}_{22} \mathrm{~N}_{4} \mathrm{O}_{6} \mathrm{Na}$ 413.1437; found $413.1445[\mathrm{M}+\mathrm{Na}]^{+}$.

\section{Methyl 3-O-methyl-2-(1H-1,2,4-triazol-1-yl-3-carbox-} amide)-2-deoxy-D-altro-hexopyranoside (4)

The foam 15 obtained in the previous preparation $(218 \mathrm{mg}$, $0.56 \mathrm{mmol}$ ) was dissolved in $30 \mathrm{ml}$ of methanol-dioxane $5 / 1$, to which was added $0.25 \mathrm{ml}$ of acetic acid. The solution was degassed by bubbling nitrogen for $10 \mathrm{~min}$, after which $250 \mathrm{mg}$ of $10 \% \mathrm{Pd}$ on carbon was added and the mixture was hydrogenated for $4 \mathrm{~h}$ on a Parr apparatus at 45 psi. The 
mixture was filtered, the filter was washed several times with hot ethanol, the volatiles were removed in vacuo and the residue was coevaporated twice with dioxane. Column purification on silica gel (gradient of $\mathrm{CH}_{3} \mathrm{OH}$ in $\mathrm{CH}_{2} \mathrm{Cl}_{2}$ 3-12\%) afforded $160 \mathrm{mg}(0.53 \mathrm{mmol}, 94 \%)$ of the title compound as a foam partially crystallizing from methanol.

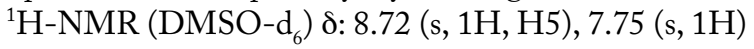
and $7.56(\mathrm{~s}, 1 \mathrm{H})\left(\mathrm{NH}_{2}\right), 5.03$ (brs, $\left.1 \mathrm{H}, 4^{\prime}-\mathrm{OH}\right), 4.96(\mathrm{~d}$, $\mathrm{J}=6.8 \mathrm{~Hz}, 1 \mathrm{H}, \mathrm{H} 1^{\prime}$ ), 4.94 (brs, 1H, 6'-OH), 4.48 (dd, 1H, $\left.\mathrm{J}_{2^{\prime}, 1^{\prime}}=6.8 \mathrm{~Hz}, \mathrm{~J}_{2^{\prime}, 3^{\prime}}=10.3 \mathrm{~Hz}, \mathrm{H} 2^{\prime}\right), 4.10\left(\mathrm{br}, 1 \mathrm{H}, \mathrm{H} 4^{\prime}\right), 3.87$ $\left(\mathrm{m}, 1 \mathrm{H}, \mathrm{H} 5^{\prime}\right), 3.83\left(\mathrm{dD}, 1 \mathrm{H}, \mathrm{J}=3.9\right.$ and $\left.10.3 \mathrm{~Hz}, \mathrm{H} 3^{\prime}\right), 3.64$ $\left(\mathrm{dAB}, 2 \mathrm{H}, \mathrm{J}=12.3 \mathrm{~Hz}, \mathrm{~J}_{5^{\prime}, 6^{\prime}}=5.9 \mathrm{~Hz}, \mathrm{H} 6^{\prime}\right), 3.24$ (s, 3H, 1'$\mathrm{OCH}_{3}$ ), 3.11 (s, 3H, 3'-OCH${ }_{3}$ );

${ }^{13} \mathrm{C}-\mathrm{NMR}\left(\mathrm{DMSO}-\mathrm{d}_{6}+\mathrm{D}_{2} \mathrm{O}\right) \delta: 160.56(\mathrm{CO}), 157.14$ (C3), 146.38 (C5), 98.98 (C1'), 77.68 (C5'), 76.01 (C3'), $63.20\left(\mathrm{C}^{\prime}\right), 60.83\left(\mathrm{C}^{\prime}{ }^{\prime}\right.$ and $\left.\mathrm{C} 2^{\prime}\right), 56.33\left(3^{\prime}-\mathrm{OCH}_{3}\right), 55.15$ $\left(1^{\prime}-\mathrm{OCH}_{3}\right)$;

ESI-MS pos.: calcd. for $\mathrm{C}_{11} \mathrm{H}_{19} \mathrm{~N}_{4} \mathrm{O}_{6}$ 303.1305; found $303.1327[\mathrm{M}+\mathrm{H}]^{+}$.

\section{Results}

Synthesis of the altritol analogue $\mathbf{1}$ and its 3'-O-methylated congener $\mathbf{2}$ is depicted in Figure 3. The route followed has been previously described for preparation of altrohexitol nucleoside monomers (Allart et al., 1999). Starting from commercially available 1,5-anhydrohexitol, ring opening of the 4,6-O-benzylidene protected allitol epoxide 5 with the 3-methylcarboxylate derivative of 1,2,4-triazole furnished the altrohexitol derivative $\mathbf{6}$ in 52\% yield. The site of alkylation proved difficult to ascertain, but a ghmbc spectrum (indicative of ${ }^{2} \mathrm{~J}$ and ${ }^{3} \mathrm{~J}$ couplings) displayed a $\mathrm{C} 5-\mathrm{H} 2{ }^{\prime}$ connectivity confining the alkyl group to either N1 or N4. As no connectivity could further be found for $\mathrm{H}_{2}^{\prime}$ with $\mathrm{C} 3$, the hexitol had to reside on N1.

Further alkylation afforded $70 \%$ of the methylated nucleoside 7. Treatment of $\mathbf{6}$ and 7, respectively, with ammonia in methanol afforded the triazolyl carboxamide analogues 9 and 10, respectively, which were deprotected with trifluoroacetic acid (TFA) to yield the target compounds 1 and 2. The small $1^{\prime}, 2^{\prime}$-coupling constants are indicative of the predominant axial orientation of the base moiety $\left({ }^{4} \mathrm{C}_{1}\right)$. Alternatively, the methyl carboxylate moiety could be preserved via direct deprotection with TFA, affording $\mathbf{8}$.

The 1'-O-methylglycosidic analogues 3 and $\mathbf{4}$ (Figure 4) could be obtained starting from ubiquitous methyl glucopyranoside, which in three steps was converted to $\mathbf{1 1}$ according to literature procedures (Neyts et al., 1996; Richtmeyer \& Hudson, 1941). Detailed NMR analysis confirmed the epoxide to possess the allo-configuration. Opening of the epoxide ring of the methyl 2,3-anhydro-allo-hexopyranoside 11 with methyl 1,2,4-triazole-3-carboxylate went more sluggishly $\left(48 \mathrm{~h}\right.$ at $\left.90^{\circ} \mathrm{C}\right)$ in comparison to opening of the epoxide 5, and afforded after careful chromatography $21 \%$ of the triazolyl congener 12 , along with $20 \%$ of the starting epoxide and $6 \%$ of the decarboxylated triazolyl derivative 13. The alkylation site could be decided on the same basis as for $\mathbf{6}$ via gHMBC NMR spectral analysis. Further treatment of $\mathbf{1 2}$ with ammonia in methanol to yield 14 and deprotection via hydrogenation afforded the target congener 3. Optionally, 12 could be further methylated with methyl iodide prior to treatment with ammonia, affording 15 in $42 \%$ yield. Notable for all NMR analyses of 12 through 15 is the high field position of the C5' signal (with $\delta$ around $58 \mathrm{ppm}$ ), which could be explained by the formation of a distorted chair conformation due to the steric hindrance of both axial substituents on $\mathrm{C}^{\prime}$ and $\mathrm{C} 3$ '.

Further deprotection of $\mathbf{1 5}$ via hydrogenation afforded the derivative 4 . The larger $6.8 \mathrm{~Hz} 1^{\prime}, 2^{\prime}$-coupling constant for the latter compounds $\mathbf{3}$ and $\mathbf{4}$, along with a large $\mathrm{J}_{2^{\prime}, 3^{\prime}}$ of $10.3 \mathrm{~Hz}$, proves the equatorial orientation of the base along with equatorially oriented methoxyl substituents, yielding a ${ }^{1} \mathrm{C}_{4}$ conformation. The steric hindrance between both methoxyl substituents precludes their axial orientation and, therefore, imposes the equato-

Figure 3. Synthetic scheme for the 1,5-anhydrohexitol analogues 1 and 2

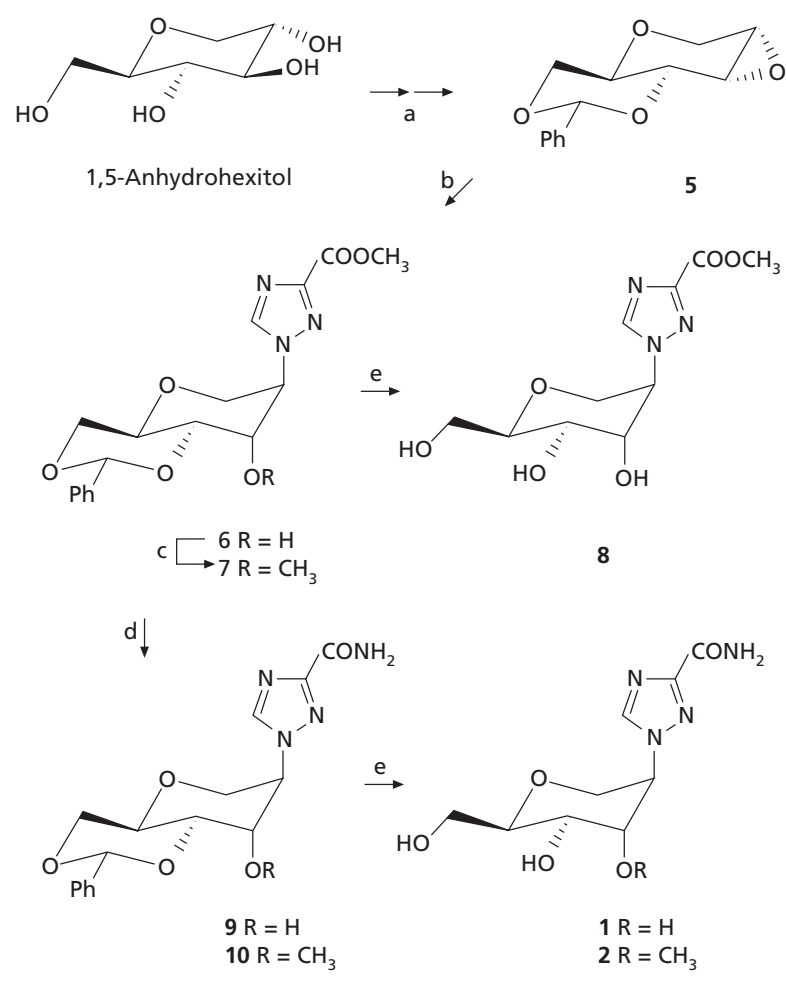

a) for synthesis of the allitol epoxide, see Allart et al., 1999; b) 1,2,4-triazole-3-methylcarboxylate 3.2 eq., 3.0 eq. NaH, DMF $90^{\circ} \mathrm{C}$ $15 \mathrm{~h}(52 \%)$; c) 1.2 eq. $\mathrm{NaH}, 30 \mathrm{~min}$. at $0^{\circ} \mathrm{C}, 1.6$ eq. $\mathrm{CH}_{3} \mathrm{I}$ slowly added, DMF, 3 h RT (70\%); d) $2 \mathrm{M} \mathrm{NH}_{3}$ in $\mathrm{CH}_{3} \mathrm{OH}$, (9: 75\%; 10: 90\%); e) $5 \%$ TFA in $\mathrm{CH}_{3} \mathrm{OH}(1: 78 \% ; 2: 71 \% ; 8: 48 \%)$. 
Figure 4. Synthetic scheme for the 1,5-anhydrohexitol analogues $\mathbf{3}$ and $\mathbf{4}$

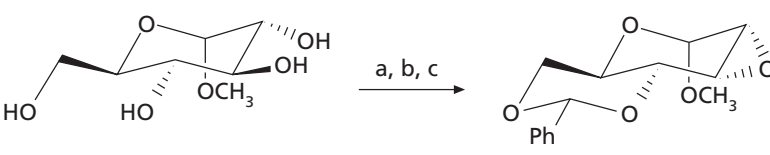

$\alpha$-D-methylglucoside
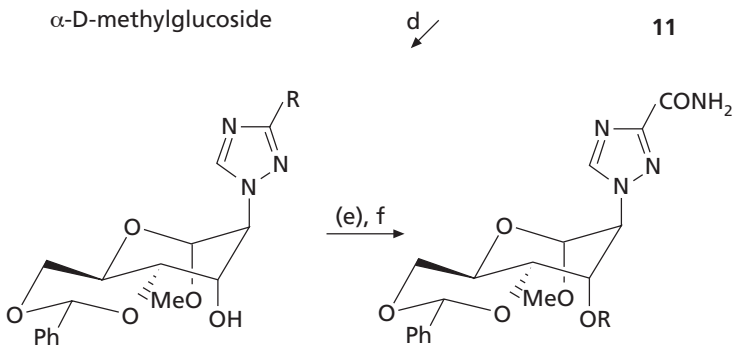

$12 \mathrm{R}=\mathrm{COOCH}_{3}$

$13 \mathrm{R}=\mathrm{H}$

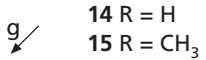

$\mathrm{HO}$

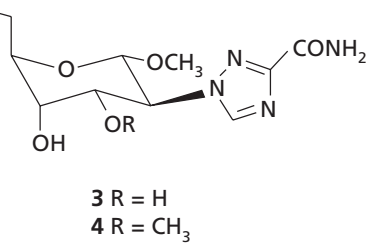

a) $\mathrm{C}_{6} \mathrm{H}_{5} \mathrm{CHO}, \mathrm{ZnCl}_{2}, 72 \mathrm{~h}(66 \%)$; b) 6 eq. $\mathrm{CH}_{3} \mathrm{C}_{6} \mathrm{H}_{5} \mathrm{SO}_{2} \mathrm{Cl}$, pyridine, $72 \mathrm{~h}$, $60^{\circ} \mathrm{C}(78 \%)$; c) $\mathrm{NaOCH}_{3}, \mathrm{CH}_{3} \mathrm{OH}, \mathrm{CH}_{2} \mathrm{Cl}_{2}(79 \%)$; d) 1,2,4-triazole-3methylcarboxylate 3.0 eq., 2.8 eq. $\mathrm{NaH}$, DMF $90^{\circ} \mathrm{C} 48 \mathrm{~h}$ (12: $21 \%$ yield was obtained along with a $20 \%$ recovery of epoxide 11 and $6 \%$ of the decarboxylated triazole 13); e) $1.2 \mathrm{eq}$. $\mathrm{NaH}, 30 \mathrm{~min}$. at $0^{\circ} \mathrm{C}$, 1.6 eq. $\mathrm{CH}_{3}$ I slowly added, DMF, $2 \mathrm{~h} \mathrm{RT}(42 \%)$; f) $2 \mathrm{M} \mathrm{NH}_{3}$ in $\mathrm{CH}_{3} \mathrm{OH}(14: 93 \%$; 15: 86\%); g) hydrogenation with $\mathrm{Pd} / \mathrm{C}, 4 \mathrm{~h}$ at 45 psi (3: $78 \% ; 4: 78 \%)$.

rial position on the base moiety, in contrast with other hexitol nucleoside analogues.

\section{Discussion}

The anhydrohexitol nucleosides with a triazolyl carboxamide base moiety 1-4 were evaluated for a possible inhibitory effect on various viruses that are (to some extent) inhibited by ribavirin. These include the pestivirus BVDV and the flavivirus YFV. Both BVDV and YFV belong, akin to $\mathrm{HCV}$, to the family of the Flaviviridae. However, unlike ribavirin, none of these compounds proved antivirally effective up to a concentration of $400 \mu \mathrm{g} / \mathrm{ml}$. Nor could any cytotoxicity be witnessed in a number of cell lines [Vero (African green monkey kidney) cells, MDBK (MadinDarby bovine kidney) cells, HEL (human embryonic lung) cells or HeLa (human cervical carcinoma epithelial) cells]. Only marginal activity was found for the 3'-O-methylated analogue 2 against BVDV [50\% effective concentration $\left(\mathrm{EC}_{50}\right)$ of $64 \mu \mathrm{g} / \mathrm{ml}$, while the $\mathrm{EC}_{50}$ value for inhibition of
BVDV and YFV replication by ribavirin is $18 \pm 5$ and 28 $\pm 18 \mu \mathrm{g} / \mathrm{ml}$, respectively. The compounds were in addition evaluated, but found inactive, against several RNA viruses (vesicular stomatitis virus, parainfluenza-3 virus, reovirus1, Sindbis virus, Coxsackie virus B4, Punta Toro virus and respiratory syncytial virus) and DNA viruses (herpes simplex virus type 1 and 2, vaccinia virus) in the appropriate cell lines (Vero, HEL or HeLa cells).

\section{Acknowledgements}

This work was supported by a grant from KU Leuven (GOA 02/13) and from the Flemish Science Foundation (G.0269.98 and G.0089.02). We thank W Van den Eynde, K Geerts, F DeMeyer and A Van Lierde for technical assistance and $\mathrm{C}$ Biernaux for editorial help.

\section{References}

Allart B, Busson R, Rozenski J, Van Aerschot A \& Herdewijn P (1999) Synthesis of protected D-altritol nucleosides as building blocks for oligonucleotide synthesis. Tetrahedron 55:6527-6546.

Balzarini J, Karlsson A, Wang L, Bohman C, Horska K, Votruba I, Fridland A, Van Aerschot A. Herdewijn P \& De Clercq E (1993) Eicar (5-ethynyl-1- $\beta$-D-ribofuranosylimidazole-4-carboxamide). Journal of Biological Chemistry 268:24591-24598.

Barnard DL (2001) Pegasys (Hoffmann La Roche). Current Opinion in Investigational Drugs 2:1530-1538.

Crotty S, Cameron C \& Andino R (2002) Ribavirin's antiviral mechanism of action: lethal mutagenesis? Journal of Molecular Medicine 80:86-95.

Crotty S, Maag D, Arnold JJ, Zhong W, Lau JY, Hong Z, Andino R \& Cameron CE (2000) The broad-spectrum antiviral ribonucleoside ribavirin is an RNA virus mutagen. Nature Medicine 6:1375-1379.

De Clercq E, Cools M, Balzarini J, Snoeck R, Andrei G, Hosoya M, Shigeta S, Ueda T, Minakawa N \& Matsuda A (1991) Antiviral activities of 5-ethynyl-1- $\beta$-D-ribofuranosylimidazole-4-carboxamide and related compounds. Antimicrobial Agents E Chemotherapy 35:679-684.

Neyts J, Reymen D, Letourneur D, Jozefonvics J, Schols D, Este J, Andrei G, McKenna P, Witvrouw M, Ikeda S, Clement J \& De Clercq E (1995) Differential antiviral activity of derivatized dextrans. Biochemical Pharmacology 50:743-751.

Neyts J, Meerbach A, McKenna P \& De Clercq E (1996) Use of the yellow fever virus vaccine strain 17D for the study of strategies for the treatment of yellow fever virus infections. Antiviral Research 30:125-132.

Richtmeyer NK \& Hudson CS (1941) Crystalline $\alpha$-methyl Daltroside and some new derivatives of D-altrose. Journal of the American Chemical Society 63:1727-1731.

Rosenfeld DA, Richtmyer NK \& Hudson CS (1948) Methyl 2,6anhydro-a-D-altroside and other new derivatives of methyl $\alpha-\mathrm{D}$ altroside. Journal of the American Chemical Society 70:2201-2206.

Scott LJ \& Perry CM (2002) Interferon- $\alpha$-2b plus ribavirin: a review of its use in the management of chronic hepatitis C. Drugs 62:507-556.

Sidwell RW, Robins RK \& Hillyard IW (1979) Ribavirin: an antiviral agent. Pharmacology \& Therapeutics 6:123-146.

Verheggen I, Van Aerschot A, Toppet S, Snoeck R, Janssen G, Claes P, Balzarini J, De Clercq E \& Herdewijn P (1993) Synthesis and 
anti-herpesvirus activity of 1,5-anhydrohexitol nucleosides. Journal of Medicinal Chemistry 36:2033-2040.

Verheggen I, Van Aerschot A, Van Meervelt L, Rozenski J, Wiebe L, Snoeck R, Andrei G, Balzarini J, Claes P, De Clercq E \& Herdewijn P (1995) Synthesis, biological evaluation and structure analysis of a series of new 1,5-anhydrohexitol nucleosides. Journal of Medicinal Chemistry 38:826-835.

Wilkinson T (2001) Hepatitis C virus: prospects for future therapies. Current Opinion in Investigational Drugs 2:1516-1522. 